\title{
Is exercise effective in reducing the risk of breast cancer in postmenopausal women?
}

McTiernan A, Kooperberg C, White E, Wilcox S, Coates R, AdamsCampbell LL, et al. Recreational physical activity and the risk of breast cancer in postmenopausal women: the Women's Health Initiative Cohort Study. JAMA 2003;290:1331-6.

Background: Heavy weight and large size have been associated with an increased risk of breast cancer. It has also been reported that regular exercise is associated with a reduced incidence of breast cancer. What is unclear is the amount or intensity of exercise required to produce this effect, and at what age the physical activity must occur.

Design: A sample of 74171 women aged 50-79 years were recruited from 40 US clinical centres between 1993 and 1998 for the Women's Health Initiative Observational Study. At study entry, information was collected on medical, reproductive, menstrual and family history as well as health behaviour, including physical activity and diet. The investigators measured height, weight and waist circumference and collected history of life-time use of hormone therapy.

Results: Breast cancer was newly diagnosed in 1780 of the women studied over a mean follow-up of 4.7 years. Women who had engaged in regular strenuous physical activity at age 35 had a $14 \%$ decreased risk of breast cancer (relative risk [RR] 0.86, $95 \%$ confidence interval [CI] 0.78-0.95) compared with less active women. A similar but nonsignificant association was observed for regular strenuous $\widehat{B}$ activity at age 18 and at age 50 . A significant trend correlating increasing levels of total current physical activity with reduced risk of breast cancer was observed in the entire study population $(p=0.03)$. When this analysis was corrected for other factors, the effect of increasing total current physical activity on risk reduction was most pronounced among women in the lowest third tertile of body mass in$\operatorname{dex}(\mathrm{BMI})(<24.1)$. The effect was also observed among those in the middle tertile of BMI (24.128.4), but the overall trend relating increasing levels of exercise with a lower risk of breast cancer was not significant in this group. Women in the highest weight category did not seem to benefit from increased physical activity.

Commentary: In this trial the strongest associations between exercise and reduced risk of breast cancer were observed among the women who weighed the least. In postmenopausal women, the most likely mechanism by which exercise might reduce the risk of breast cancer is the reduction of body fat, which leads to reduced substrate for the production of estrogen from androgen in fat tissue through aromatization. Physical activity may also increase levels of sex hormone-binding globulin and thereby reduce the amount of estradiol in the free, most biologically active state. Exercise may also reduce levels of growth factors such as insulin, which is known to act as a mitogen. Interestingly, the reduction in risk associated with exercise was seen among both women who did and those who did not take hormone replacement therapy.

Practice implications: This article is one of several reporting an association between physical activity and breast cancer prevention. ${ }^{1,2} \mathrm{It}$ provides the additional evidence, however, that current exercise and exercise engaged in after menopause are both associated with a decreased risk of breast cancer. The effects seem to be realized even with a relatively small amount of exercise (the equivalent of 1.25-2.5 hours per week of brisk walking) and are similar to those associated with reductions in the risk of cardiovascular disease among postmenopausal women and of diabetes among subjects with impaired glucose tolerance.,

\section{Kathleen I. Pritchard}

Clinical Trials and Epidemiology

Toronto Sunnybrook Regional Cancer Centre and Department of Medicine

University of Toronto

Toronto, Ont.

\section{References}

1. Friedenreich CM. Physical activity and cancer prevention: from observational to interventional research. Cancer Epidemiol Biomarkers Prev 2001;10:287-301.

2. McTiernan A, Stanford JL, Weiss NS, Daling JR, Voigt, LF. Occurrence of breast cancer in relation to recreational exercise in women age 50-64 years. Epidemiology 1996;7:598-604.

3. Manson JE, Greenland P, LaCroix AZ, Stefanick ML, Mouton CP, Oberman A, et al. Walking compared with vigorous exercise for the prevention of cardiovascular events in women. $N$ Engl 7 Med 2002;347:716-25.

4. Knowler WC, Barrett-Connor E, Fowler SE, Hamman RF, Lachin JM, Walker EA, et al. Reduction in the incidence of type 2 diabetes with lifestyle intervention or metformin. $N$ Engl $\mathcal{F}$ Med 2002;346:393-403. 